\title{
Significados de bienestar subjetivo en la elección de carrera: una realidad de los estudiantes de Comunicación y de Periodismo de la Universidad de Colima
}

\author{
Beatriz Paulina Rivera Cervantes
}

\begin{abstract}
Resumen
La calidad de vida de los seres humanos ha sido estudiada, con mayor ahínco, en las últimas décadas. Las mayores necesidades de consumo; la posesión de bienes y su exhibición ante los demás, parecieran ser lo más importante para la gran mayoría de jóvenes, o al menos eso se percibe en redes sociales como Instagram y Facebook. No obstante, cada día es más común escuchar a estudiantes de nivel superior, acerca de sentirse ansiosos o deprimidos. En ese contexto, la posibilidad de sentirse satisfecho ante la vida, o tener bienestar subjetivo, podría limitarse, mucho más, cuando se toman decisiones. La poca demanda en México en los programas de comunicación, respecto a hace veinte años, también repercutió en la Universidad de Colima, donde se ofertan dos licenciaturas, Periodismo y Comunicación. El presente texto muestra resultados de un estudio cualitativo, realizado con la técnica del grupo de discusión, que tuvo como objetivo el identificar las diferencias y semejanzas en la elección de carrera, por género, de los estudiantes de Comunicación y de Periodismo de la Universidad de Colima, para conocer la incidencia de la capacidad de agencia en el bienestar subjetivo de los estudiantes.
\end{abstract}

Abstract

In the last few decades, life quality has been studied with greater diligence. High consumption needs; possession of goods and the need to show them, seem to be the most important thing for the vast majority of young people, or at least that is perceived in social networks such as Instagram and Facebook. However, every day it is more common to hear higher level students, feeling anxious or depressed. In this context, the possibility of feeling satisfied with life, or having subjective well-being, could be much more limited when decisions are made. The low demand that communication programs have in Mexico, compared to twenty years ago, also had an impact on the University of Colima, where two degrees are offered, Journalism and Communication. This text presents the results of a qualitative study, carried out with a discussion group technique with Communication and Journalism students from the University of Colima, which aimed to identify the differences and similarities in the career choice, it was made by gender, in order to know the incidence of agency capacity on the subjective well-being of students.

Palabras Clave

Agencia, Autonomía, Elección de carrera, Bienestar subjetivo.

Keywords

Agency, Autonomy, Career choice, Subjective well-being

\section{Introducción}

Ante el creciente interés en el estudio global de la calidad de vida, del bienestar subjetivo y del desarrollo humano, gracias a la Organización para la Cooperación y el Desarrollo
Económicos (OCDE), así como a las aportaciones teóricas de Ryff (2012); González (2014); Yamamoto (2015); Temkin (2016); Millán y Castellanos (2018), pero, sobre todo, gracias a la Teoría del Desarrollo Humano desde el enfoque de las capacidades, de Amartya Sen (2000), colaborador vital de 
los informes anuales de Desarrollo Humano del Programa de las Naciones Unidas para el Desarrollo (PNUD), y a las contribuciones de Nussbaum (2012) a dicha teoría, hoy en día se cuenta con fundamentos científicos para la medición de procesos subjetivos, como el bienestar, que pretenden incidir en las políticas públicas de las naciones.

Aunado a ello, Rosanna Reguillo (2013), indica que ser joven, en la actualidad mexicana, es difícil porque las oportunidades para ingresar a la universidad son bajas y hay pocos empleos de calidad, además de que los índices de pobreza y violencia van en aumento; dichos factores "hacen parte de la experiencia cotidiana y subjetiva de millones de jóvenes" (Reguillo, comunicación personal, 1 de septiembre de 2013). Por lo tanto, afirma la autora, en algunos jóvenes se presenta un desencanto en su subjetividad, lo cual ha orillado a que este sector de la población quiera recuperar su capacidad de agencia.

La agencia, esa capacidad propia para actuar, para generar cambios, es un derecho individual en el que Sen (2000) asegura que se es libre cuando una persona alcanza todo aquello que valora y cuando no hay otros sujetos que interfieran en sus decisiones de vida, por lo que sus procesos de elección son inmunes ante los demás y se convierten en sujetos agentes. Por su parte, Ian Gough (2007), también concibe a esta habilidad o capacidad de elección y toma de decisiones como de suma importancia, sin embargo, la materializa como autonomía. Esto conlleva el ser capaz de formular objetivos, saber cómo alcanzarlos y evaluar el éxito de este proceso. No obstante, el análisis del desarrollo de la capacidad de agencia para lograr el bienestar subjetivo, en los jóvenes, al momento de tomar decisiones importantes en la vida, como la carrera profesional, ha sido poco estudiado.

Reguillo (2013) y Saraví (2015) coinciden en la detección de experiencias de desigualdad social, cultural y subjetiva en los jóvenes mexicanos. Ambos autores afirman que este sector de la población no ve a la educación como sinónimo de progreso o ascenso social. En contraparte, Rojas (2018) menciona que la satisfacción ante la vida aumenta cuando se tiene mayor grado de estudios, de acuerdo con los resultados de la investigación realizada por Imagina México, A.C., en 2012, aplicada en 100 municipios de México, con la finalidad de indagar sobre el bienestar subjetivo de los ciudadanos del país.
De acuerdo con Universia (2014), en la Encuesta Nacional de Juventud (ENJ), de los 36 millones de jóvenes mexicanos participantes en dicho estudio, el 15\% aceptó estudiar en una universidad porque no tuvo otra opción. En este caso, la libertad de elegir o de ejercer su capacidad de agencia se pone en tela de juicio y, en muchos casos, la deserción universitaria se hace evidente. Esta misma red de universidades -Universiareportó que en nuestro país sólo el $25 \%$ de los estudiantes universitarios concluyen sus estudios, principalmente porque las instituciones de educación superior no cumplían con las expectativas de los alumnos y porque el centro de estudios no era el deseado (Universia, 2014).

De entre los países miembros de la OCDE, México ocupó en 2014 el primer lugar en tasa de homicidios, con 18 por cada 100000 habitantes y Colima fue la entidad más violenta en el primer semestre de 2019 (Proceso, 2019). Ante ello, sentirse seguro, pleno o satisfecho con la vida, puede verse como complicación, ya que el contexto es un factor extrínseco que puede influir en la felicidad, es decir, en la dimensión del bienestar subjetivo, la cual alude a lo que los ciudadanos sienten cuando evalúan la vida en su conjunto, de acuerdo a sus experiencias y sentimientos que van generando con sus vivencias.

Por otra parte, en el ciclo escolar 2017-2018, Colima fue el segundo estado en el país con más mujeres matriculadas en una carrera universitaria, según el Panorama de la Educación Superior por Entidad realizado en 2018 por la Dirección General de Educación Superior Universitaria (DGESU), de la Secretaría de Educación Pública (SEP). Dicha población representa al 53\% de 28,180 personas que estudiaban en el ciclo escolar mencionado. Estos datos van en sintonía con las aportaciones de Sen (2000), quien indica que el poder que las mujeres han ganado, en las últimas décadas, influye positivamente en el desarrollo de su país. El poder está relacionado con la educación, las oportunidades de empleo y las actitudes que la familia y la sociedad tienen respecto a estas circunstancias.

Entre las carreras profesionales con mayor demanda en México, hace 30 años, estaban las del área de comunicación y de periodismo, cuya oferta educativa se amplió a más de mil programas educativos en todo el país, con presencia en todos los estados de la República Mexicana (Fuentes Navarro, 2018). Aun así, explica el autor, "la formación universitaria de 
comunicadores en México parece sufrir desde hace décadas de hipertrofia, aunque la disyuntiva relevante en términos académicos esté y haya estado siempre en la calidad y en la pertinencia" (p. 12). En ese sentido, la Universidad de Colima, a través de la Facultad de Letras y Comunicación no ofrece un sólo programa de estudios de esta disciplina, sino dos licenciaturas por separado; desde 2003, la de Comunicación y la de Periodismo, y, desde entonces, la carrera con mayor demanda ha sido la de Comunicación.

Por lo general los espacios disponibles en cada proceso de admisión, en el programa de Periodismo, no son ocupados al cien por ciento, por lo cual, y como parte de la política de inclusión, en dicha institución el promedio del bachillerato solicitado para este programa es de cinco décimas más bajo que el de Comunicación, sin embargo, sigue sin igualarse la demanda entre ambos programas.

Además, ya que se obtienen los resultados del proceso de admisión, en la Universidad de Colima y con la finalidad de brindar espacios a más personas y mantener la mayor cobertura educativa, la institución facilita que a un programa de poca demanda, como Periodismo, se inscriban estudiantes que no eligieron dicha carrera como primera opción. No obstante, se pone sobre la mesa la posibilidad de que los indicadores académicos de competitividad, como el del egreso, se afecten dado que tan sólo en 2018, la tasa fue de $18.18 \%$ (Facultad de Letras y Comunicación, 2018).

De cara a este panorama desolador, se determinó realizar un estudio cualitativo, con el objetivo de identificar las diferencias y semejanzas en la elección de carrera, por género, de los estudiantes de Comunicación y de Periodismo de la Universidad de Colima, para conocer la incidencia de la capacidad de agencia en el bienestar subjetivo de los estudiantes.

\section{Revisión de literatura}

Para comprender el fenómeno a estudiar, así como la relación entre sus conceptos básicos, la presente investigación cualitativa contempló la revisión documental de distintos enfoques teóricos, en donde el análisis del objeto de estudio se centró en dos categorías: la capacidad de agencia en la elección de carrera y el bienestar subjetivo, los cuales se presentan a continuación.
Como se ha mencionado, la importancia del estudio del bienestar subjetivo cobró mayor auge en las últimas décadas, a partir de la creación del Programa de las Naciones Unidas para el Desarrollo (PNUD) y de la OCDE, con el propósito de aminorar la pobreza mundial, a través del impulso de instrumentación que facilitara la medición del desarrollo humano. Por ello, y para los fines de esta pesquisa, se retomaron las aportaciones de Sen (2000), mediante la Teoría del Desarrollo Humano desde el enfoque de las capacidades. Ahí, el autor explica que el individuo crece como ser humano al tener opciones y libertades para realizar lo que se propone y ser quien desea ser, a través del acceso a la educación, a la salud, a la longevidad, a la participación social y a la dignidad.

Asimismo, Sen define al desarrollo humano a través de tres elementos: las libertades, las capacidades y la agencia. Por lo tanto, la libertad cobra gran peso en la perspectiva del bienestar subjetivo y del desarrollo humano, en esta teoría. Al respecto, el autor indica que la libertad individual y el desarrollo social están estrechamente relacionados porque las oportunidades que brindan las instituciones están condicionadas a la libertad que sienten los individuos "para participar en las decisiones sociales y en la elaboración de las decisiones públicas que impulsan el progreso de estas oportunidades" (Sen, 2000, p.4).

Esta concepción para alcanzar el desarrollo humano, coincide con la visión de Rojas (2005), y de la OCDE (2011), respecto al bienestar subjetivo, mismo que se concibe como la manera en que un sujeto experimenta sus condiciones de vida, según su marco de referencia o contexto. Consecuentemente, el bienestar subjetivo forma parte esencial de los seres humanos para la construcción de igualdad, de progreso y de calidad de vida.

Para caracterizar y medir la agencia, como una de las capacidades primordiales que contempla la Teoría del Desarrollo Humano desde el enfoque de capacidades, se consideró útil emplear el constructo de la autonomía de un individuo, debido a su funcionalidad al momento de medir los niveles de apropiación de la misma. Fueron Len Doyal e Ian Gough (1994), a través de la Teoría de las Necesidades Humanas, quienes desarrollaron la concepción de la agencia bajo el término autonomía. Dicha teoría explica que la actuación y la responsabilidad de los individuos conllevan a poseer capacidades físicas y mentales para hacerlo. En 
ese sentido, se requiere de procesos como la deliberación y la elección y, para realizarlas, es importante contar con la autonomía mencionada, término explicado como "la habilidad para hacer elecciones y tomar decisiones informadas sobre lo que debería hacerse y cómo hacerlo" (Gough, 2007, p. 186).

Existen tres niveles de autonomía en la Teoría de las Necesidades Humanas de Doyal y Gough (1994). El primero, vinculado a la capacidad cognitiva y emocional, debido a que el raciocinio y la responsabilidad deben estar presentes cuando se toman decisiones (Gough, 2007). Por lo tanto, se explica que se tiene un grado mínimo de autonomía cuando se tiene confianza en sí mismo para actuar socialmente; de igual manera se tiene cuando se es capaz de comunicar cuáles objetivos se ha planteado un individuo y este se percibe con la habilidad de conseguirlos, además de que asume la responsabilidad del resultado de las acciones elegidas.

El segundo nivel de autonomía es cuando se comprende el individuo, a sí mismo, como un ser cultural; cuando comprende a esta como parte de su contexto y cuando sabe lo que este espera de él como sujeto autónomo. Gough (2007) señala que para alcanzar este segundo nivel de autonomía es necesario desarrollar el aprendizaje para toda la vida, con el apoyo de profesores en la educación formal.

El tercer nivel de autonomía implica lo propuesto, también, por Sen, las oportunidades. Para estar en posibilidad de actuar, es necesario ejercitar la capacidad de elección y esta se dará cuando existan opciones reales para realizar actividades con gran significado para un sujeto, ya que como menciona Gough (2007), "una mínima libertad de actuación es un componente esencial de la autonomía de actuación en todas las culturas" (p. 187). En ese sentido, aprender a identificar las oportunidades es un ejercicio continuo que con el tiempo debe irse fortaleciendo si se desea aumentar el nivel de autonomía.

La Teoría de las Necesidades Humanas establece que una vez asumidos los tres niveles de autonomía anteriores, se alcanza otro nivel todavía superior: la autonomía crítica. En este punto se comparan reglas culturales, se analizan reglas de la propia cultura y se adoptan reglas de otras. Para ello, un sujeto requiere de libertades, tanto de actuación, como política para deliberar crítica y creativamente sobre la vida que elige tener (Gough, 2007), aunque debe contemplar cuatro precondiciones sociales: la producción cultural, la reproducción de esta, su transmisión y la autoridad política.
En este tenor, la autonomía no sólo es un factor individual, sino también, social, al estar vinculada, necesariamente, al tipo de sociedad en que se encuentra inmerso un individuo. Por lo tanto, puede concluirse que, al tener autonomía crítica, se aprende a resolver problemas, conscientemente y es mucho más sencillo contar con bienestar subjetivo.

\section{Método}

Dado que el presente texto se deriva de un proyecto de investigación concluido y mucho más amplio, para fines prácticos, se muestran aquí los resultados de ocho de los 16 grupos de discusión que contempló en total el estudio.

El diseño metodológico de la investigación se planteó a partir de la pregunta: ¿cuáles son las diferencias y semejanzas entre la elección de carrera, por género y por trayectoria escolar, de los estudiantes de Comunicación y de Periodismo de la Universidad de Colima, que permiten conocer la incidencia de la capacidad de agencia en su bienestar subjetivo? Para dar respuesta a ella, la metodología empleada fue la cualitativa, y se optó por realizar el estudio con un enfoque fenomenológico, mediante los grupos de discusión, debido a que facilitó la obtención de elementos reflexivos por parte de los informantes y permitió explicar la esencia de las experiencias de los participantes. Por ende, se obtuvieron significados valiosos para comprender lo que sucede en este contexto social y cultural específico.

Para Ibáñez (1992), el alcance epistemológico de esta técnica de investigación cualitativa es de gran aliento, porque a través de la interacción discursiva se detectan consensos y tal como explica Chávez (2004), con la dirección de un moderador, los grupos de discusión permiten que los sujetos sean libres para explicar el sentido y el significado que otorgan a sus acciones cotidianas, con lo que es posible configurar categorías para el análisis de un problema a partir del discurso generado. Además, Chávez enfatiza que los grupos de discusión son una vía práctica para "observar el proceso de producción y reproducción de la ideología de un grupo social al que pertenecen los participantes" (2004, p. 232).

Los ocho grupos de discusión se dividieron en dos, los de Comunicación y los de Periodismo, y a la vez, se subdividieron en hombres y mujeres; a partir de ellos, se 
crearon cuatro grupos de segundo semestre y otros cuatro de octavo (véase Tabla1), con la intención de comparar los significados de una decisión recientemente tomada (la elección de la carrera profesional), en el primer año de estudios, y la que debería estar afianzada, en el último año de la carrera.

La finalidad de separar a los hombres de las mujeres, fue para obtener información con perspectiva de género -y no de sexo-, debido a que existen autores que definen la perspectiva de género como un enfoque construido socialmente (Goffman, 1977) y como una construcción discursiva (Butler, 2007), a partir del comportamiento y el discurso de los seres humanos. La conformación descrita de los grupos se hizo para vigilar epistemológicamente la investigación y permitió cierta representatividad semántica de la población de estudio.

La unidad de análisis fueron los estudiantes universitarios, ya que, de ellos se buscó obtener información valiosa sobre sus características; mientras que la unidad de observación fueron los estudiantes de las licenciaturas en Periodismo y en Comunicación de la Facultad de Letras y Comunicación de la Universidad de Colima, puesto que, a nivel nacional, los planes de estudio de ambos Programas Educativos (PE), suelen ofertarse en uno sólo, por lo que la situación en la Universidad de Colima es muy particular, a diferencia de los del resto del país.

El estudio se diseñó para que la unidad de información estuviera representada por una muestra seleccionada para obtener los datos requeridos. En la preproducción se invitó a cinco participantes voluntarios para cada uno de los grupos $\mathrm{y}$, finalmente, 37 sujetos participaron en dichos grupos, como se muestra en la Tabla1. De esta manera, y como menciona Ibáñez (1992), se aseguró que la producción del discurso tuviera el contexto lingüístico adecuado para el uso social de los resultados y, en consecuencia, el diseño de la investigación otorgó la confiabilidad al estudio comparativo.
Número de participantes volunta 1.

Clave del grupo Comunicación Periodismo

Primer año de estudios

\begin{tabular}{|c|c|c|c|}
\hline \multirow[t]{4}{*}{ Hombres } & $17.05 .2019 / \mathrm{COM} / \mathrm{H} / 1 \mathrm{~A}$ & 5 & 5 \\
\hline & Último año de estudios & \multirow{3}{*}{5} & \multirow{3}{*}{4} \\
\hline & & & \\
\hline & 09.05.2019/COM/H/4A & & \\
\hline \multirow{6}{*}{ Mujeres } & Primer año de estudios & \multirow{3}{*}{5} & \multirow{3}{*}{3} \\
\hline & & & \\
\hline & 13.05.2019/COM/M/1A & & \\
\hline & Último año de estudios & \multirow{3}{*}{4} & \multirow{3}{*}{6} \\
\hline & & & \\
\hline & 08.05.2019/COM/M/4A & & \\
\hline
\end{tabular}

Fuente: Elaboración propia

Para diseñar el instrumento se contempló la estructura enunciativa del campo semántico, el cuál giró en torno al sentido que la autonomía tiene en la elección de carrera de los estudiantes, y así, darle orden a las categorías correspondientes que generaría la guía de los cinco detonadores que desligaron la información necesaria para su análisis posterior. En la Tabla 2 presentan las categorías de análisis y las dimensiones del estudio que dieron pie a la guía empleada en todos los grupos de discusión. 
Matriz de categorías de ana 2.

\begin{tabular}{|c|c|c|c|}
\hline $\begin{array}{l}\text { Categorías } \\
\text { de } \\
\text { análisis }\end{array}$ & Dimensiones & Observación & . \\
\hline \multirow[t]{4}{*}{$\begin{array}{l}\text { Niveles } \\
\text { de } \\
\text { autonomía }\end{array}$} & $\begin{array}{l}\text { Capacidad } \\
\text { cognitiva } \\
\text { emocional }\end{array}$ & $\begin{array}{l}\text { Capacidad } \\
\text { intelectual } \\
\text { para plantear } \\
\text { objetivos. }\end{array}$ & \multirow[t]{4}{*}{$\begin{array}{l}\text { Teoría de las } \\
\text { necesidades } \\
\text { humanas } \\
\text { (Doyal y } \\
\text { Gough, 1994). }\end{array}$} \\
\hline & & $\begin{array}{l}\text { Confianza } \\
\text { suficiente } \\
\text { para actuar y } \\
\text { participar en la } \\
\text { vida social al } \\
\text { elegir la carrera. } \\
\text { Responsabilidad } \\
\text { sobre su elección } \\
\text { de carrera }\end{array}$ & \\
\hline & $\begin{array}{l}\text { Grado } \\
\text { comprensión } \\
\text { cultural que una } \\
\text { persona tiene } \\
\text { sobre sí misma, } \\
\text { sobre su cultura } \\
\text { y sobre lo que } \\
\text { se espera de ella } \\
\text { como individuo } \\
\text { dentro de la } \\
\text { misma. }\end{array}$ & $\begin{array}{l}\text { Comprensión de } \\
\text { sí mismo como } \\
\text { ser cultural. }\end{array}$ & \\
\hline & Las oportunidades & $\begin{array}{l}\text { Capacidad } \\
\text { para identificar } \\
\text { oportunidades } \\
\text { valiosas. }\end{array}$ & \\
\hline \multirow[t]{2}{*}{$\begin{array}{l}\text { Elección } \\
\text { de } \\
\text { carrera }\end{array}$} & $\begin{array}{l}\text { Oportunidades de } \\
\text { las instituciones. }\end{array}$ & $\begin{array}{l}\text { Aspiraciones de } \\
\text { formación en la } \\
\text { Universidad de } \\
\text { Colima. }\end{array}$ & $\begin{array}{l}\text { PNUD } \\
\text { (González, } \\
2014)\end{array}$ \\
\hline & $\begin{array}{l}\text { Confianza en las } \\
\text { instituciones. }\end{array}$ & & \\
\hline \multirow[t]{2}{*}{$\begin{array}{l}\text { Bienestar } \\
\text { subjetivo }\end{array}$} & $\begin{array}{l}\text { Posibilidad de } \\
\text { participar e influir } \\
\text { en la sociedad. }\end{array}$ & $\begin{array}{l}\text { Participación en } \\
\text { organizaciones } \\
\text { (compromiso } \\
\text { con el entorno, } \\
\text { sentimiento de } \\
\text { capacidad para } \\
\text { transformar el } \\
\text { entorno). }\end{array}$ & \multirow[t]{2}{*}{$\begin{array}{l}\text { Teoría del } \\
\text { desarrollo } \\
\text { humano desde } \\
\text { el Enfoque de } \\
\text { Capacidades } \\
\text { (Sen, 2000 } \\
\text { y Nussbaum, } \\
\text { 2012) }\end{array}$} \\
\hline & $\begin{array}{l}\text { Tener y desarrollar } \\
\text { un proyecto de } \\
\text { vida propio. }\end{array}$ & $\begin{array}{l}\text { Evaluación de } \\
\text { la consistencia } \\
\text { con el proyecto } \\
\text { de vida. (Lo } \\
\text { que quiero ser } \\
\text { y lo que se ha } \\
\text { logrado) }\end{array}$ & \\
\hline
\end{tabular}

Fuente: Elaboración propia
Posteriormente, y para interpretar la información recabada en los grupos de discusión, se realizó un análisis argumentativo, basado en el estudio comparativo del listado de juicios lógicos obtenidos del discurso, es decir, de los consensos grupales, se identificaron los temas o grandes tópicos de los que se habló, llamados por Giménez (1989), objetos discursivos, así como también se definió lo que se dijo de ellos, los predicados. Después de ese proceso de análisis, se elaboraron los esquemas comparativos que facilitaron la comprensión y explicación del fenómeno analizado, mediante el ordenamiento de los juicios lógicos, respecto al mayor número de consensos detectados hasta enlistar el menor número de estos.

\section{Resultados}

Para dar respuesta a la pregunta de investigación, a continuación se muestran los principales hallazgos, primeramente, de los estudiantes del primer año de estudios $\mathrm{y}$, posteriormente, los del último año de ambas carreras profesionales.

En los consensos de los juicios lógicos de los estudiantes del primer año de Comunicación, se detectó que la responsabilidad fue uno de los elementos importantes para ambos grupos de Comunicación (hombres y mujeres), lo cual indica que tanto ellos, como ellas, identifican el rol que les espera como comunicadores. No obstante, fueron las mujeres quienes detallaron más cuáles son las expectativas que la sociedad esperará que ellas cubran, profesionalmente hablando. Asimismo, se observó que ambos grupos identifican sus oportunidades de crecimiento, sobre todo, en función de la seguridad que expresaron en sus discursos, al saber qué es lo que más disfrutan de la Comunicación, por lo tanto, ambos grupos cuentan con sus tres niveles de autonomía.

Como se observa en el Cuadro 3, respecto a la felicidad y su relación con los proyectos personales y profesionales, que sirvieron para analizar el bienestar subjetivo de los estudiantes, se identificaron consensos relativos al disfrute de la carrera por lo que en ella se aprende, sin embargo, los hombres del primer año de Comunicación tienen metas precisas y, al mismo tiempo, están presionados por cumplirlas, lo cual dista de las mujeres, quienes hablaron de la satisfacción de todo el proceso de aprendizaje y no dejan la felicidad como 
una meta lejana, sino por el contrario, para ellas, esta es y la sienten inmediata. En cambio, los hombres esperan hasta tener resultados, a largo plazo, para sentir dicha satisfacción, sobre todo, porque, para ellos, el reconocimiento de terceros está de por medio, en este caso, el de sus padres, por lo tanto, la satisfacción interna de ellos no está autorregulada, y, de esta manera, el bienestar subjetivo está mucho más posicionado en el discurso de las mujeres que en el de los hombres.

Tabla 3.

Consensos de hombres y mujeres del primer año de estudios de la licenciatura en Comunicación sobre el bienestar subjetivo

\begin{tabular}{l}
\hline El bienestar subjetivo \\
\hline Mujeres \\
$13.05 .2019 / \mathrm{COM} / \mathrm{M} / 1 \mathrm{~A}$ \\
\hline Es el enriquecimiento \\
y crecimiento como \\
persona para aportar a \\
los demás. \\
Es ganarse las cosas \\
por esfuerzo propio y \\
porque se merecen.
\end{tabular}

Consensos Es disfrutar y gozar grupales al aprender, valorar y conocer una profesión sin calificaciones perfectas.

Es la gratificación del ahora a pesar del sacrificio económico por vivir fuera de casa. Es sentirse satisfecho con lo que se va logrando. Hombres 17.05.2019/COM/H/1A Está condicionada por las expectativas que cada quién se crea y estas ponen presión sobre ti.

Se relaciona con la presión que uno mismo se establece respecto a metas y a la presión de los padres al querer ver resultados sobre la educación recibida.

Es hacer lo que te gusta.

Es un proceso reflexivo.

Es seguir estudiando para hacer proyectos sin complicaciones.

Es querer a tu carrera por su sentido humanista y altruista.

Consensos Es hacer lo que les gusta, en este caso, generales estudiar Comunicación, lo cual es motivo de satisfacción..

Fuente: Elaboración propia.

Al haber analizado comparativamente a ambos grupos del primer año de Comunicación, se concluye que la elección de la carrera incide positivamente en el bienestar subjetivo, sobre todo, en las mujeres, puesto que cuentan con sus tres niveles de autonomía, y por consiguiente, se deduce que ellas tienen mayor posibilidad de arraigar su capacidad de agencia en la toma de decisiones.

Por su parte, en los dos grupos, de hombres y de mujeres, del primer año de estudios en Periodismo, se identificaron pocos consensos generales; únicamente coincidieron en que los valores universales deben ser puestos en práctica para ejercer el periodismo con compromiso. Las mujeres saben quiénes son como individuos, inmersas en su contexto y tienen muy claro cuál es el papel que fungen en el sentido profesional. Ellos sólo realizaron críticas de la situación del periodismo, más no señalaron cómo se observan profesionalmente hablando, por el contrario, denotan que la responsabilidad de saber quiénes deben ser como periodistas debe recaer en terceros, más no se aprecia un compromiso por parte de ellos, como futuros profesionistas. Por consiguiente, los dos primeros niveles de autonomía, de la Teoría de las Necesidades Humanas de Doyal y Gough (1994), están presentes en las mujeres, pero no en los hombres del primer año de estudios de la licenciatura en Periodismo.

Ahora bien, al analizar el discurso sobre el bienestar subjetivo, mostrado en la Tabla 4, se detectó que ellas hablaron de hacer funcionar las cosas cuando los planes no se concretan, como el estar inscritas en una carrera que no fue su primera opción profesional, que, coincidentemente, era su caso. Por ende, su capacidad de agencia y sus niveles de autonomía sí están explícitamente ligados a su bienestar subjetivo, a diferencia de los hombres, quienes no llegaron a este tipo de consensos. 
Tabla 4.

Consensos de hombres y mujeres del primer año de estudios de la licenciatura en Periodismo sobre el bienestar subjetivo

\begin{tabular}{l}
\hline El bienestar subjetivo \\
\hline Mujeres \\
28.05.2019/PER/M/4A \\
\hline Es estar en el lugar \\
correcto en el \\
momento correcto \\
y si no es así, uno lo \\
hace correcto. \\
Es luchar por seguir los \\
sueños personales.
\end{tabular}

Consensos Es ver más allá de grupales lo económico, y ver a la profesión como un estilo de vida y no como un trabajo.

Es prepararse porque la mediocridad no es para uno.

Hombres

29.05.2019/PER/H/4A

Es plantearse retos, pero viviendo el presente.

Es que la carrera te permita ser creativo para escribir historias o tomar fotografías para compartirlas.

No es la perfección, sino estar bien, con vida y salud.

Es dejarse llevar, pero echándole ganas para salir adelante.

Es encontrar a las Es a largo plazo porque personas correctas para no importa a qué se enfocarse en lo que a dedique uno, dónde uno le gusta. esté o cuánto gane porque el proyecto de vida no se vincula con el Periodismo.

Está marcada por el destino.

Es disfrutar el proceso estudiantil.

Es tener un trabajo seguro, pero los profesores dicen que la profesión no ayuda a ello.

No debería depender de otras personas.

Consensos Es luchar por seguir los sueños personales, al generales plantearse retos, pero viviendo el presente.

Fuente: Elaboración propia.

Ellos, señalaron que desean vivir el presente, al dejarse llevar, sin proyectos de vida concretos, aunque, el aspecto familiar o amoroso no estuvo presente en los discursos, ni de las mujeres, ni de los hombres del primer año de estudios en Periodismo.
Debido a que el camino para llegar a ese primer año de estudios es distinto, entre ambos programas educativos es notorio que fueron las mujeres quienes lograron sobreponerse ante las adversidades. En ambas carreras, ellas indicaron buscar orientación en sus profesores y en sus padres, aunque, principalmente, el discurso de las estudiantes del primer año de Periodismo indica que su actitud ante la vida, es de lucha, asertividad y adaptabilidad. Términos como conformismo o mediocridad, fueron mencionados, en el sentido de evitarlos para alcanzar su felicidad, mientras que en el de los hombres, se percibe implícitamente, pero como parte del fracaso que algunos dijeron sentir por no estudiar la carrera que querían, inicialmente. Por lo tanto, de los cuatro grupos de discusión, los hombres del primer año de Periodismo tienen menor autonomía en su capacidad de agencia y son menos propensos a alcanzar su bienestar subjetivo.

En relación a los estudiantes del último año de estudios en Comunicación, el discurso reflejó que las mujeres vislumbran más que los hombres las posibles rutas de crecimiento que les brinda la carrera de Comunicación. Aun así, ambos grupos valoran las oportunidades, que han tenido, respecto a la vinculación con el sector productivo y por las redes de colaboración que fueron creando, con apoyo de profesores, a lo largo de casi cuatro años. Es notorio, en la Tabla 5, que han sabido reconocer la parte social e individual para alcanzar un alto nivel de autonomía, y que se perciben más cercanos a su bienestar subjetivo. 
Tabla 5.

Consensos de hombres y mujeres del primer año de estudios de la licenciatura en Comunicación sobre el bienestar subjetivo

\begin{tabular}{|c|c|c|}
\hline \multicolumn{3}{|c|}{ El bienestar subjetivo } \\
\hline & Mujeres & Hombres \\
\hline & $08.05 .2019 / \mathrm{COM} / \mathrm{M} / 4^{\mathrm{a}}$ & 09.05.2019/COM/H/4A \\
\hline \multirow{10}{*}{$\begin{array}{l}\text { Consensos } \\
\text { grupales }\end{array}$} & $\begin{array}{l}\text { Es explorar las } \\
\text { capacidades } \\
\text { profesionales. }\end{array}$ & $\begin{array}{l}\text { Es hacer lo que te gusta } \\
\text { aunque te paguen poco. }\end{array}$ \\
\hline & Es generar contenido & Se encuentra \\
\hline & $\begin{array}{l}\text { adecuado para los } \\
\text { demás. }\end{array}$ & $\begin{array}{l}\text { hay satisfacción en las } \\
\text { labores que se realizan. }\end{array}$ \\
\hline & $\begin{array}{l}\text { Es poder hacer algo por } \\
\text { los demás. }\end{array}$ & $\begin{array}{l}\text { Es lo opuesto a la } \\
\text { rutina. }\end{array}$ \\
\hline & $\begin{array}{l}\text { No se vincula con el } \\
\text { dinero }\end{array}$ & $\begin{array}{l}\text { Se relaciona con la } \\
\text { diversidad de áreas que }\end{array}$ \\
\hline & & $\begin{array}{l}\text { ofrece la carrera de } \\
\text { Comunicación. }\end{array}$ \\
\hline & Es sentirse útil. & Se cumple con las \\
\hline & & metas alcanzadas \\
\hline & & $\begin{array}{l}\text { que se trazaron en } \\
\text { bachillerato. }\end{array}$ \\
\hline & \multicolumn{2}{|l|}{ Da seguridad. } \\
\hline $\begin{array}{l}\text { Consensos } \\
\text { generales }\end{array}$ & \multicolumn{2}{|c|}{$\begin{array}{l}\text { Se vincula con la diversidad de áreas que } \\
\text { ofrece la carrera de Comunicación y se } \\
\text { encuentra cuando hay satisfacción en las } \\
\text { labores que se realizan, más no por el dinero } \\
\text { obtenido con estas. }\end{array}$} \\
\hline
\end{tabular}

Los hallazgos en los dos grupos del último año de estudios de la licenciatura en Periodismo fueron muy distintos a los de Comunicación y a los del primer año de estudios en ambas carreras. Los hombres expresaron mayor satisfacción con lo vivido en su proceso formativo, aunque realizaron críticas hacia la labor del periodista, por los problemas que identificaron del ejercicio de esta profesión. Sin embargo, evaluaron positivamente el trabajo en equipo; el fogueo que el plantel les brindó y las oportunidades, en general, que les otorgó la Universidad de Colima. El discurso de las mujeres estuvo cargado de pesimismo y negatividad, al rescatar pocos aspectos positivos respecto a su satisfacción con la elección de su carrera.

Al revisar la Tabla 6, y durante la situación discursiva de las mujeres, se detectó que sus proyectos de vida son casi nulos y estos van acompañados de temores y angustia, situación que no se presentó en el grupo de los hombres. Ante ello, Rojas expresa que los "estudiantes felices no sólo tienen una mejor disposición al aprendizaje y una menor propensión a desertar, sino que, sobre todo, estarán siendo felices en el presente y adquiriendo el conocimiento para ser felices en el futuro" (2018, p. 75$)$, por lo tanto, se puede hablar de que las mujeres del último año de estudios, en Periodismo, no están cercanas a la agencia que se requiere para alcanzar el bienestar subjetivo.

Tabla 6.

Consensos de hombres y mujeres del primer año de estudios de la licenciatura en Comunicación sobre el bienestar subjetivo

\begin{tabular}{|c|c|c|}
\hline \multicolumn{3}{|c|}{ El bienestar subjetivo } \\
\hline & Mujeres & Hombres \\
\hline & 28.05 .2019 & 29.05.2019/ \\
\hline & /PER/M/4A & $/ \mathrm{H} / 4 \mathrm{~A}$ \\
\hline \multirow{7}{*}{$\begin{array}{l}\text { Consensos } \\
\text { grupales }\end{array}$} & $\begin{array}{l}\text { Significa tener el } \\
\text { control. }\end{array}$ & $\begin{array}{l}\text { Se relaciona con el } \\
\text { Periodismo Deportivo. }\end{array}$ \\
\hline & $\begin{array}{l}\text { Es tener un trabajo } \\
\text { estable. }\end{array}$ & $\begin{array}{l}\text { Se relaciona con la } \\
\text { producción de medios. }\end{array}$ \\
\hline & $\begin{array}{l}\text { Es lo opuesto a } \\
\text { institucionalizar la } \\
\text { vida. }\end{array}$ & $\begin{array}{ll}\text { Se da con los } \\
\text { compañeros } & \text { de } \\
\text { trabajo adecuados. } & \end{array}$ \\
\hline & Se vincula & Es tener trabajo. \\
\hline & $\begin{array}{l}\text { la producción } \\
\text { radiofónica. }\end{array}$ & \\
\hline & & $\begin{array}{l}\text { Es estudiar } \\
\text { posgrado. }\end{array}$ \\
\hline & & $\begin{array}{l}\text { Tiene que ver con la } \\
\text { compañía. }\end{array}$ \\
\hline
\end{tabular}

Consensos Se relaciona con la producción de medios de generales comunicación.

Fuente: Elaboración propia.

Lo más sobresaliente al comparar los resultados del último año de estudios, en ambas carreras, fueron las discrepancias entre las mujeres. Mientras que las de Comunicación ven que su carrera las hace felices, a pesar de las adversidades mencionadas por ellas, como el poco reconocimiento social de su profesión o el salario bajo que podrían tener, las mujeres de Periodismo expresaron temor por no llegar a tener el control de su vida e institucionalizar su vida. Por su argumentación, se comprende que buscan la emancipación, más que la expansión de la libertad, mencionada por Sen (2000), como medio principal de desarrollo y fin primordial del ser humano. 


\section{Conclusiones}

El bienestar subjetivo se da cuando un individuo experimenta y valora sus propias experiencias de vida, respecto a diferentes situaciones (Millán y Castellanos, 2018) y, de acuerdo con el Cuadro 6, las mujeres de Periodismo fueron las únicas que no expresaron sentir felicidad o satisfacción cuando hablan de ejercer su carrera profesional, sino que resaltaron la incertidumbre que se genera en torno al ejercicio periodístico. Tampoco mencionaron que los aspectos familiares, de amistad o amorosos se relacionaran con su felicidad, o si tienen algún proyecto en concreto, lo cual es preocupante, ya que, al momento de realizar el estudio, estaban a un par de meses de egresar de su licenciatura. Por lo tanto, se determinó, a partir de los resultados, que el tener una trayectoria escolar más avanzada, en el área de Periodismo no es sinónimo de autonomía o de una capacidad de agencia desarrollada.

Cuando la autonomía es baja, las personas sienten la presión de demandas, estándares, reglas y expectativas externas, además de sentir que están a la merced de dichas expectativas. La autonomía es muy difícil de alcanzar para personas que están acostumbradas al control externo y que dependen del mismo para su autoestima. (Pick, Sirkin, Ortega, Osorio, Martínez, Xocolotzin Givaudan, 2007, p. 226)

Esta visión de la vida, (en la que no hay valoraciones positivas sobre su bienestar subjetivo) tiene una conexión con el estilo de vida que desean tener los llamados millenials, quienes nacieron cercanos a la fecha en que inició este nuevo milenio y que son, justamente, los jóvenes del sector que participaron en el estudio. De acuerdo con Llantada (2017), ellos buscan tener experiencias y no la posesión de objetos o servicios de lujo, porque quieren hacer lo que les gusta, sin embargo, no siempre definen esos gustos, por lo que se hace presente la frustración en sus vidas. Parte de la explicación del comportamiento de este segmento de edad se da porque, según Llantada, los padres de los millenials los acostumbraron a darles satisfactores inmediatos, por lo cual, sus prácticas culturales digitales, de los últimos años están asociadas a la estimulación y aprobación en las redes sociales (nuevamente, inmediatas), que los hacen tomar decisiones en función de dicha estimulación. Sería riesgoso generalizar y decir que estas condiciones aplican al cien por ciento en este grupo en particular. Lo que sí es importante mencionar, es que a pesar de los temores y las experiencias negativas que dijeron pasar como estudiantes, las alumnas optaron por continuar en ese proyecto llamado licenciatura, porque identificaron que había aprendizajes que podrían aplicar para su vida laboral, aunque esta no fuera dentro del periodismo, lo cual es igualmente preocupante para el campo de estudios de la enseñanza de la Comunicación y del Periodismo, en general.

La significación otorgada a la elección de la carrera de Comunicación es asertiva y no hay discrepancias en cuanto a perspectiva de género se refiere. No obstante, sí hay diferencias en los hombres y las mujeres estudiantes de Periodismo. Asimismo, su agencia personal no los encamina al bienestar subjetivo y los costos para la profesión pueden ser muy altos, especialmente, porque estudiar esta carrera profesional no contribuye al poder ganado por las mujeres, ya mencionado por Sen (2000). En el campo de la Comunicación y del Periodismo siempre son bien recibidas las discusiones académicas, en cuanto a la pertinencia de su oferta o a la reputación de esta profesión. Sin embargo, pesquisas en todo Iberoamérica siguen surgiendo, ante entre las disparidades del propio ejercicio periodístico, así como de la labor del comunicador del Siglo XXI. Es imperante fomentar una vocación auténtica y en beneficio de los futuros profesionistas y para la sociedad que demanda a gritos estrategias eficaces que permitan el desarrollo humano y así tener comunidades justas, equitativas, con armonía y, por ende, productivas, ya que, como Fuentes Navarro menciona "el entorno en el que sucede la comunicación, en un sentido y en otro, podrá transformarse en la dirección en que la impulsen no sólo sus profesionales, sino sus agentes sociales" (2018, p. 17), pero paralelamente, el presente trabajo demuestra que no se puede ser un agente social, sin antes desarrollar la agencia personal.

Ahora se tiene la seguridad de que la formación de comunicadores en el país debe continuar sus esfuerzos por conocer a los estudiantes de hoy, así como sus necesidades. Esta investigación demuestra que la elección de su profesión y el ejercicio de la misma, tienen significados distintos en hombres y mujeres, debido a que, los comunicadores tienen la encomienda de articular competencias profesionales para interpretar el sentido de la realidad social, a través de su capital cultural, y, así, diseñar, ejecutar y evaluar, proyectos como verdaderos agentes sociales y todo indica que, la identidad 
el comunicador es mucho más clara para los estudiantes. En cambio, para la formación de periodistas se requieren mayores estudios epistemológicos que avalen la evolución del propio periodismo y ampliar los estudios acerca de su propia enseñanza dentro de las aulas, ante el creciente fenómeno de producción y consumo de desinformación digital, por ejemplo, que ha puesto de cabeza a nuestra nueva ecología, y, por supuesto, habría que tener más discusiones para definir a quién se le atañe dicha responsabilidad, si al campo de la Comunicación o al del Periodismo, o si dichas carreras deben permanecer en simbiosis constante, en su oferta educativa, para aminorar el poco bienestar subjetivo identificado en programas de estudio como el de Periodismo, en la Universidad de Colima.

Esta situación educativa, cultural y profesional, inmersa en un pasaje de pluralidad de valores para la integración social, todavía tiene grandes retos para encaminar a los estudiantes hacia su bienestar subjetivo y, aunque el significado de este siga siendo un proceso intrínseco, el campo académico de la Comunicación y las instituciones de Educación Superior de nuestro país, pueden brindar más oportunidades para afianzar la elección de la carrera profesional de los jóvenes, y así, colaborar en el alcance de los objetivos de desarrollo sostenible que el mundo demanda, en el cual los profesionistas saben cuál es su lugar, a pesar de las complejidades de las realidades emergentes del siglo XXI.

\section{Referencias}

Bauman, Z. (2016, noviembre, 7). Entrevista a Zygmunt Bauman: Bauman: . En el mundo actual todas las ideas de felicidad acaban en una tienda"(Gonzalo Suárez, entrevistador). Recuperado de: https://www.elmund o.es/papel/lideres/2016/11/07/58205c8ae5fdeaed768b 45d0.html?fbclid=IwAR0DmXuMXHltQCEsERpccK C1D96wNVkijis6cc-oKqtgS6-HaJ3nVeS4B1U

Bedoya, C. (2010). Amartya Sen y el desarrollo humano. Revista Nacional de la Investigación. Memorias. 1 (13), 277-288.

Butler, J. (2007). El género en disputa. El feminismo y la subversión de la identidad. Barcelona: PAIDÓS.

Castellanos, R. (2016). "El bienestar subjetivo como enfoque e instrumento de la administración pública y las políticas públicas". David Gómez-Álvarez y Víctor Ortiz. (Comp.) El bienestar subjetivo en América Latina. México: Universidad de Guadalajara.
Castro Solano, A. (2010). Fundamentos de psicología positiva. Buenos Aires: Paidós 89. Colecc. Evaluación Psicológica.

Chávez, M. (2004). De cuerpo entero... Todo por hablar de música. Reflexión técnica y metodológica del grupo de discusión. México: Universidad de Colima.

Dirección General de Educación Superior Universitaria (2018). Panorama de la Educación Superior por Entidad. México: Secretaría de Educación Pública. Recuperado de: http://www.dgesu.ses.sep.gob.mx/Panorama_de_1 a_educacion_superior.aspx

Doyal, L. y Gough, I. (1994). Teoría de las necesidades humanas. Madrid: Icaria.

Facultad de Letras y Comunicación (2018). Informe de Labores 2018. México: Universidad de Colima.

Fuentes Navarro, R. (2018). "Coyunturas y disyuntivas de los estudios y las prácticas de Comunicación: hacia una perspectiva regional.” En León, G., Castillo, E., Montes, M. y Oliveros, M. (Coord.). Tendencias críticas y nuevos retos de la Comunicación en México. México: Qartuppi. Recuperado de: http://www.qartuppi.com/2018/TEND ENCIAS.pdf

Giménez, G. (1989). Poder, estado y discurso. Perspectivas sociológicas y semiológicas del discurso político-jurídico. México: UNAM.

Goffman, E. (1977). The arrangement between the sexes. Theory and society. 4 (3). 301-331.

González, P. (2014). Bienestar subjetivo integral, capacidades y políticas públicas. Realidad, datos y espacio. Revista Internacional de Estadística y Geografía. 5, (1). 4-15.

Gough, I. (2007). El enfoque de las capacidades de M. Nussbaum: un análisis comparado con nuestra teoría de las necesidades humanas. Papeles de Relaciones Ecosociales y Cambio Global. CIP-Ecosocial/Icaria. 100, 176-202.

Hernández Sampieri, R., Fernández Collado, C., y Baptista Lucio, P. (2006). Metodología de la investigación (4th ed.). México: McGraw-Hill.

Ibáñez, J. (1992). Más allá de la sociología. El grupo de discusión: técnica y crítica. España: Siglo XXI.

Llantada, A. (2017). Millennials vs Generación X: las dos son un dolor de cabeza. ¿Cómo llegó a suceder esta diferencia tan abismal entre las actitudes de ambas generaciones? Entrepreneur. Recuperado el 22 de julio de 2019 de: https://www.entrepreneur.com/article/296 104

Millán, R. y Castellanos, R. (2018). Bienestar subjetivo en México. México: UNAM.

Nussbaum, M. (2012). Las mujeres y el desarrollo humano. El enfoque de las capacidades. Barcelona: Herder.

OCDE (2013). México. Panorama de la Educación 2013: Indicadores de la OCDE. Recuperado de: http://www.oe cd.org/education/Mexico_EAG2013\%20Country\%20n 
ote\%20(ESP).pdf?fbclid=IwAR3xmNoiCEKQuqePyS ayNa53JT7s-Ib5M4lnrqMzEkF22NuoytrpG59eSD0

OCDE (2017). ¿Cómo va la vida en México? Recuperado el 2 de diciembre de 2018 de: https://www.oecd.org/statist ics/Better-Life-Initiative-country-note-Mexico-in-Es pagnol.pdf

OCDE (2017). La Lucha por la Igualdad de Género. Una Batalla Cuesta Arriba. ¿Cómo se compara México?. Recuperado el 9 de enero de 2019 de: https://www.oecd .org/mexico/Gender2017-MEX-es.pdf

Pick, S., Sirkin, J., Ortega, I., Osorio, P., Martínez, R., Xocolotzin, U. Givaudan, M. (2007). Escala Para Medir Agencia Personal y Empoderamiento (ESAGE). Revista Interamericana de Psicología/Interamerican Journal of Psychology. 41 (3), 295-304.

Proceso (2019). "Primer semestre de 2019 registra 15 mil 51 asesinatos; Colima, la entidad más violenta”. Proceso. Recuperado de: https://www.proceso.com.mx/593290/ primer-semestre-de-2019-registra-15-mil-51-asesina tos-colima-la-entidad-mas-violenta

Reguillo, R. (2013, septiembre, 1). Entrevista a Rossana Reguillo: Jóvenes, sociedad digital y política (C. Scolari, entrevistador). Recuperado de: https://hipermediaciones .com/2013/09/01/entrevista-a-rossana-reguillo-jovene s-sociedad-digital-y-politica/

Reguillo, R. (2013). Jóvenes en la encrucijada contemporánea: en busca de un relato de futuro. Debate Feminista, 48. Núm. C, 137-151. doi: 10.1016/S0188-9478(16)30092-5. Recuperado de: http://www.elsevier.es/es-revista-debate-feminista-37 8-articulo-jovenes-encrucijada-contemporanea-busca -un-S0188947816300925

Ryff, C. (2012). Existential well-being and health. In P.T.P. Wong (ed), The human quest for meaning. (pp. 233-348). New York: Routledge.

Rojas, M. (2005). El bienestar subjetivo en México y su relación con indicadores objetivos. Consideraciones para la política pública. En Garduño, L., Salinas, B. y Rojas, M. (Coord.) Calidad de vida y bienestar subjetivo en México. (pp. 83-111). México: Universidad de las Américas de Puebla/Centro de Estudios sobre Calidad de Vida y Desarrollo Social/International Society of Quality of Life Studies/Plaza y Valdés.

- (2014). El estudio científico de la felicidad. México: Fondo de Cultura Económica.

- (2018). "Educación, capital humano y felicidad". Bienestar subjetivo en México. México: Instituto de Investigaciones Sociales de la UNAM.

Saraví, G. (2015). Juventudes fragmentadas. Socialización, clase y cultura en la construcción de la desigualdad. México: FLACSO - CIESAS.

Temkin, B. (2016). The Negative Influence of Labor Informality on Subjective Well-Being. Global Labour Journal. 7. Doi 10.15173/glj.v7i1.2545.
Universia (2014). Jóvenes mexicanos dejan la universidad porque ésta no cumple con sus expectativas. Recuperado de: http://noticias.universia.net.mx/en-portada/noticia /2014/12/08/1116582/jovenes-mexicanos-dejan-univer sidad-esta-cumple-expectativas.html

Yamamoto, J. (2015). Un modelo de bienestar subjetivo para Lima Metropolitana. (Tesis doctoral). Perú: Pontificia Universidad Católica del Perú. 